\title{
Các nhân tố ảnh hưởng đến ý định khởi nghiệp của sinh viên Trường Đại học Nam Cần Thơ
}

\section{Factors affecting entrepreneurship intentions of Nam Can Tho university students}

\author{
Nguyễn Văn Định ${ }^{1 *}$, Lê Thị Mai Hương ${ }^{2}$, Cao Thị Sen ${ }^{2}$ \\ ${ }^{1}$ Trường Đại học Nam Cần Thơ, Việt Nam \\ ${ }^{2}$ Trường Đại học Tây Đô, Việt Nam \\ *Tác giả liên hệ, Email: hoangdinh670@gmail.com
}

\begin{abstract}
THÔNG TIN
TÓM TẮT

DOI: $10.46223 / \mathrm{HCMCOUJS}$.

Nghiên cứu nhằm xác định "Các nhân tố ảnh hưởng đến ý định khởi nghiệp của sinh viên Trường đại học Nam Cần Thơ.” Phương pháp thực hiện đề tài là định tính để xác định mô hình nghiên cứu và

Ngày nhận: 02/11/2020

Ngày nhận lại: 19/03/2021

Duyệt đăng: 12/05/2021

Tù khóa:

doanh nghiệp; khởi nghiệp; sinh viên định lượng để xác định và đo lường mức độ ảnh hưởng của các nhân tố đến ý định khởi nghiệp. Dữ liệu nghiên cứu được thu thập từ kết quả khảo sát trực tiếp bằng bảng câu hỏi với 310 sinh viên năm ba và năm tư của Khoa Kinh tế và Khoa Kiến trúc - Xây dựng và Môi trường. Kết quả nghiên cứu mô hình cấu trúc tuyến tính (SEM) cho thấy có 05 nhân tố tác động đến ý định khởi nghiệp của sinh viên theo mức độ giảm dần là: đặc điểm tính cách; thái độ đối với hành vi khởi nghiệp; môi trường giáo dục; nhận thức kiểm soát hành vi và nguồn vốn. Bên cạnh đó, kết quả cũng đã chỉ ra chuẩn chủ quan không có tác động đến ý dịnh khởi nghiệp của sinh viên. Từ kết quả nghiên cứu, nhóm tác giả đề xuất các hàm ý quản trị nhằm nâng cao ý định khởi nghiệp của sinh viên Trường Đại học Nam Cần Thơ trong thời gian tới.
\end{abstract}

Keywords:

businesses; start-ups; students

\section{ABSTRACT}

The study aims to determine "Factors affecting entrepreneurship intentions of Nam Can Tho University students." The method of implementing the topic is qualitative to determine the research model and quantitative to determine and measure the influence of factors on entrepreneurial intentions. Research data is collected from direct survey results with questionnaires with 310 3rd and 4th year students of the Faculty of Economics and Faculty of Architecture - Construction and Environment. The results of the study of the linear structural model (SEM) show that there are 05 factors affecting the students' entrepreneurial intention in decreasing order: personality characteristics; attitude towards entrepreneurial behavior; educational environment; perceived behavioral control and capital. In addition, the results also show that the subjective norm has no impact on the students' intention to start a business. From the research results, the authors propose managerial implications to improve the entrepreneurial intention of students of Nam Can Tho University in the coming time. 


\section{Giới thiệu}

Xu hướng khởi nghiệp là một trong những động lực thúc đẩy sự phát triển các sản phẩm công nghệ sáng tạo đáp ứng yêu cầu của đời sống xã hội. Tinh thần khởi nghiệp là một trong những giải pháp cơ bản góp phần thúc đẩy tăng trưởng kinh tế, giải quyết vấn đề việc làm. Khởi nghiệp là chìa khóa quan trọng để tăng trưởng kinh tế, việc thúc đẩy giới trẻ khởi nghiệp là một trong những ưu tiên hàng đầu của các nhà chính sách (Shapero \& Sokol, 1982). Tại Việt Nam, vấn đề khởi nghiệp kinh doanh được thực hiện trên tất cả lĩnh vực và mọi đối tượng nói chung và trong sinh viên đang học tại các trường đại học nói riêng. Theo báo cáo của Ngân hàng Phát triển Châu Á (ADB) cho biết tỷ lệ thanh niên Việt Nam thất nghiệp năm 2020 có thể lên tới 13.2\% (Thu Hang, 2020). Do đó, trong bối cảnh việc làm là khan hiếm so với với số lượng sinh viên tốt nghiệp thì giải pháp cấp thiết hiện nay để giảm lượng sinh viên tốt nghiệp thất nghiệp đó là khơi dậy tinh thần khởi nghiệp kinh doanh. Để hoạt động này được triển khai rộng khắp trong các cơ sở giáo dục đại học, Bộ Giáo dục và Đào tạo đã ban hành Quyết định số 1230/QĐ-BGDĐT ngày 30 tháng 03 năm 2018 về việc ban hành Kế hoạch triển khai đề án "Hỗ trợ học sinh sinh viên khởi nghiệp đến năm 2025" (Bộ Giáo dục và Đào tạo, 2018). Tuy nhiên, để đạt được mục tiêu đến năm 2020 cả nước có 5,000 Doanh nghiệp khởi nghiệp đổi mới sáng tạo và thành phố Cần Thơ phải có 13,800 doanh nghiệp hoạt động cần phải có sự chung tay góp sức của cộng đồng, trong đó vai trò của các trường đại học và sinh viên hết sức quan trọng (My Thanh, 2017). Trường Đại học Nam Cần Thơ (DNC) cũng không nằm ngoài quỹ đạo đó, nhà trường luôn đề cao tinh thần khởi nghiệp của sinh viên. Với mô hình doanh nghiệp trong trường đại học được phát triển mạnh mẽ, đồng thời nhà trường phối hợp với các doanh nghiệp để tổ chức ngày hội việc làm, hội thảo về khởi nghiệp; tổ chức các cuộc thi nhằm phát huy tinh thần khởi nghiệp, giúp sinh viên đẩy mạnh tính năng động, nhận thức kinh doanh, đổi mới sáng tạo và bản lĩnh kinh doanh. Nhằm nâng cao tinh thần kinh doanh của sinh viên khi còn đang học ở giảng đường đại học thì cần xác định được các nhân tố ảnh hưởng đến ý định khởi nghiệp của sinh viên. Do đó, nghiên cứu "Các nhân tố ảnh hưởng đến ý định khởi nghiệp của sinh viên trường đại học Nam Cần Thơ" nhằm tạo cơ sở khoa học cho việc đề xuất hàm ý quản trị nâng cao ý định khởi nghiệp cho sinh viên.

\section{Cơ sở lý thuyết và mô hình nghiên cứu}

\subsection{Cơ sở lý thuyết và các nghiên cúu có liên quan}

Ý định khởi nghiệp có thể được định nghĩa là sự chuẩn bị của một cá nhân để bắt đầu một doanh nghiệp (Souitaris, Zerbinati, \& Al-Laham, 2007); chỉ ra việc lập kế hoạch và tạo dựng một doanh nghiệp là một quá trình (Gupta \& Bhawe, 2007). Để tạo lập doanh nghiệp của riêng mình thì cá nhân phải bắt nguồn từ việc họ nhận ra cơ hội, tận dụng các nguồn lực sẵn có (Kuckertz \& Wagner, 2010). Theo Schwarz, Wdowiak, Almer- Jarz, và Breitenecker (2009), dự định khởi sự kinh doanh của sinh viên xuất phát từ các ý tưởng của sinh viên và được định hướng đúng đắn từ chương trình giáo dục và những người đào tạo. Trong nghiên cứu này ý định khởi nghiệp kinh doanh của sinh viên là tiền đề, là sự sẵn sàng thực hiện hành vi kinh doanh có chủ ý của sinh viên với mong muốn tạo ra sản phẩm, dịch vụ có giá trị nhằm đáp ứng nhu cầu của xã hội.

Theo thuyết hành động hợp lý (TRA), hành vi được quyết định bởi dự định thực hiện hành vi và khả năng kiểm soát của họ (Ajzen \& Fishbein, 1975). Thuyết (TRA) được sử dụng để dự báo hành vi tự nguyện và giúp đỡ những người khác trong việc nhận ra yếu tố tâm lý của mình. Hai yếu tố chính ảnh hưởng đến ý định là thái độ cá nhân và chuẩn chủ quan. Trong đó, thái độ của một cá nhân đối với hành vi thể hiện mức độ đánh giá tiêu cực hoặc tích cực của cá nhân đối với hành vi. Thái độ chịu ảnh hưởng của giá trị mong đợi của cá nhân. Chuẩn chủ quan thể hiện sự liên quan đến nhận định của người khác (gia đình, bạn bè) cảm thấy như thế nào khi cá nhân thực hiện hành vi đó. Trên cơ sở thuyết hành động hợp lý của Ajzen và Fishbein (1975), Ajzen (1991) 
phát triển Thuyết hành vi hoạch định (TPB) để dự báo và làm sáng tỏ hành vi con người trong một bối cảnh cụ thể. Nhân tố thứ ba, có ảnh hưởng đến ý định của con người là yếu tố nhận thức kiểm soát hành vi. Nhân tố này đã chỉ ra sự dễ dàng hay khó khăn khi thực hiện hành vi, nhận thức của cá nhân là do khả năng của bản thân và nguồn lực để thực hiện hành vi (Ajzen, 1991). Trong đó, dự định khởi nghiệp kinh doanh là một yếu tố có trước, quyết định việc thực hiện hành vi kinh doanh. Nó sẽ cho phép dự đoán cả những hành vi không hoàn toàn điều khiển được với giả định một hành vi có thể được dự báo hoặc được giải thích bởi dự định để thực hiện hành vi đó (Kolvereid, 1996).

Nghiên cứu của Ngo và Cao (2016), dự định khởi nghiệp của sinh viên được phân loại theo 03 hướng tiếp cận là: Chương trình giáo dục và ý định khởi nghiệp của sinh viên; môi trường và ý định khởi nghiệp; Dự định khởi nghiệp của một cá nhân bao gồm (giới tính, tính cách, tư duy và thái độ). Bên cạnh đó, kết quả nghiên cứu của Truong và Nguyen (2019) dựa trên việc tổng hợp các công trình nghiên cứu trong và ngoài nước về ý định khởi nghiệp của sinh viên đã khái quát được 07 nhân tố tác động gồm: Kỳ vọng của bản thân; thái độ với khởi nghiệp; năng lực bản thân cảm nhận; cảm nhận về tính khả thi; chuẩn mực niềm tin; vốn tri thức và vốn tài chính.

Sinh viên Trường Đại học Kỹ thuật Công nghệ Cần Thơ với ý định khởi nghiệp kinh doanh đã cho thấy có 07 nhóm nhân tố chính ảnh hưởng đến hành vi khởi nghiệp của sinh viên bao gồm: đặc điểm tính cách; thái độ cá nhân; nhận thức và thái độ; giáo dục khởi nghiệp; nhận thức điều khiển hành vi; quy chuẩn và thái độ và quy chuẩn chủ quan (T. A. Phan \& Tran, 2017). Theo L. K. Le (2018), ý định khởi nghiệp của sinh viên khối ngành kinh tế chịu ảnh hưởng bởi 05 nhân tố: yếu tố bên trong của sinh viên (nghị lực, tự tin, đam mê) và yếu tố bên ngoài (nguồn vốn, chính sách hỗ trợ của trường). Mặt khác, theo T. N. D. Le và Nguyen (2019), ý định khởi nghiệp của sinh viên ngành Du lịch và Quản trị Kinh doanh ảnh hưởng bởi 04 nhân tố: môi trường giáo dục; mục tiêu và hoài bão kinh doanh của sinh viên; nhận thức kiểm soát hành vi và tính hấp dẫn của việc khởi nghiệp; trong đó nhận thức kiểm soát hành vi là nhân tố tác động mạnh nhất. Tuy nhiên, trong nghiên cứu cũng đã chỉ ra chuẩn chủ quan, nhu cầu và nguồn vốn không có ảnh hưởng đến ý dịnh khởi nghiệp của sinh viên. Theo Chau và Huynh (2020), ý định khởi nghiệp của sinh viên bị ảnh hưởng mạnh nhất về môi trường khởi nghiệp, tiếp đó là giáo dục khởi nghiệp ở trường đại học; nhận thức kiểm soát hành vi; chuẩn chủ quan; xu hướng chấp nhận rủi ro và sự tự tin.

Ngoài ra, ý định khởi nghiệp của sinh viên được xác định bởi kết quả phân tích mô hình cấu trúc tuyến tính (SEM) của V. Q. Phan và Trac (2020) là: thái độ cá nhân, chuẩn mực chủ quan và kiểm soát nhận thức hành vi. Hơn nữa, ý định khởi nghiệp của sinh viên còn được khẳng định qua sự tự tin về tính khả thi là yếu tố quan trọng để đi đến ý định khởi nghiệp. Nghiên cứu về các yếu tố ảnh hưởng đến ý định khởi nghiệp cho thấy có mối quan hệ tích cực giữa hoạt động giảng dạy, hoạt động ngoại khóa, ý kiến của những người xung quanh cũng như sở thích kinh doanh của mỗi cá nhân có tác động tích cực đến sự tự tin về tính khả thi là yếu tố trung gian hình thành ý định khởi nghiệp (H. T. Nguyen \& Nguyen, 2016). Dù vậy, nghiên cứu chưa phân tích về các yếu tố bên trong (đặc điểm tính cách, thái độ cá nhân, nhận thức kiểm soát hành vi và nguồn vốn) để khởi nghiệp.

\subsection{Giả thuyết và mô hình nghiên cứu}

\subsection{1. Đặc điểm tính cách}

Đặc điểm tính cách nói lên tính cách của một cá nhân thể hiện xu hướng chọn những nghề nghiệp đòi hỏi sự khám phá, sáng tạo. Sinh viên khao khát có một địa vị trong xã hội hoặc muốn được thể hiện, muốn được tôn trọng và biết đến càng nhiều. Xu hướng sẵn sàng chấp nhận rủi ro trong kinh doanh là một nét trong đặc điểm tính cách của sinh viên (Bui, Le, Dao, \& Nguyen, 
2011). Theo Wilbard (2009), đã cô đọng lại thành 05 đặc điểm cá nhân mà mỗi nhà khởi nghiệp đều có, bao gồm: sự tự tin; sự năng động nhạy bén; có hoài bão; khuynh hướng tự chủ cao và sẵng sàng chấp nhận rủi ro. Đặc điểm tính cách ảnh hưởng đến nhu cầu mong muốn thành công, sự tự tin và khả năng của bàn thân, thể hiện sự sẵn sàng chấp nhận rủi ro và tác động tích cực đến mong muốn và sự tự tin khởi sự kinh doanh (Scott, 1991). Đặc điểm tính cách cũng đã được khẳng định trong các nghiên cứu của (Ngo \& Cao, 2016; T. A. Phan \& Tran, 2017) có tác động đến ý định sinh viên khởi nghiệp.

\section{H1: Đặc điểm tính cách ảnh hưởng thuận chiều đến sụ tụ tin về tính khả thi khởi nghiệp}

\subsubsection{Thái độ đối với hành vi khởi nghiệp}

Theo Ajzen (1991), thái độ đối với hành vi khởi nghiệp thể hiện sự đánh giá tích cực hay tiêu cực, ủng hộ hay phản đối của một cá nhân về hành vi dự định thực hiện. Khi sinh viên có thái độ hứng thú với việc khởi nghiệp kinh doanh, nhận thấy lợi ích và khi có cơ hội và nguồn lực sẽ tiến hành khởi nghiệp. Các yếu tố khác ảnh hưởng đến thái độ đối với hành vi kinh doanh như tâm thế chấp nhận rủi ro, sự tự do, độc lập (Krueger, Reilly, \& Carsrud, 2000). Trong nghiên cứu này, đó là sự đánh giá tích cực hay tiêu cực, ủng hộ hay phản đối của một người có dự định đối với hành vi kinh doanh mà họ hướng tới. Thái độ đối với hành vi khởi nghiệp đã được khẳng định có ảnh hưởng tích cực đến ý định khởi nghiệp của sinh viên trong các nghiên cứu của (D. T. T. Le \& Nguyen, 2016; T. N. D. Le \& Nguyen, 2019; T. A. Phan \& Tran, 2017; V. Q. Phan \& Trac, 2020; Truong \& Nguyen, 2019).

H2: Thái độ đối với hành vi khởi nghiệp ảnh hưởng thuận chiều đến sụ tụ tin về tính khả thi khơi nghiệp

\subsubsection{Nguồn vốn}

Nhân tố được cho là quan trọng trong việc hiện thực hóa ý tưởng kinh doanh để cá nhân triển khai hoạt động kinh doanh vào trong thực tiễn đó là nguồn vốn. Quá trình để tiếp cận được với ưu đãi tài chính vẫn là một hành trình vô cùng gian nan đối với các doanh nghiệp khởi nghiệp (Dong Nghi \& Thien Minh, 2018). Khi khởi nghiệp kinh doanh, chỉ có một số ít người có đủ vốn để mở doanh nghiệp, còn đa số cần phải huy động vốn từ các nguồn khác nhau để khởi nghiệp. Hầu hết các doanh nhân trẻ đều sử dụng tài trợ của cha mẹ, anh em và bạn bè trong giai đoạn đầu khởi nghiệp, đây là nguồn tài chính quan trọng nhất (Q. Le, 2007). Nguồn vốn kinh doanh đã được khẳng định có ảnh hưởng tích cực đến ý định khởi nghiệp của sinh viên trong các nghiên cứu của (L. K. Le, 2018; T. N. D. Le \& Nguyen, 2019; Truong \& Nguyen, 2019).

\section{H3: Nguồn vốn ảnh hưởng thuận chiều đến sự tụ tin về tính khả thi khởi nghiệp}

\subsubsection{Nhận thức kiểm soát hành vi}

Theo Ajzen (1991), nhận thức kiểm soát hành vi là đề cập đến nhận thức của cá nhân về mức độ dễ dàng hay khó khăn; có bị kiểm soát, hạn chế hay không khi thực hiện hành vi. Nó có thể được đánh giá qua cảm nhận của cá nhân về khả năng tồn tại, phát triển của doanh nghiệp khi khởi nghiệp, khả năng thành công khi kinh doanh, những kiến thức, kinh nghiệm cá nhân hay khả năng tiếp cận thông tin để làm cho việc khởi nghiệp trở nên khả thi. Một cá nhân có tiềm năng ý định khởi nghiệp phải có mong muốn và nhận thấy tính khả thi của việc ý định khởi nghiệp (Shapero \& Sokol, 1982). Đây là khái niệm gần với khái niệm về năng lực cá nhân của (Bandura, 1997); khái niệm về sự tự tin trong mô hình (SEE) của Shapero và Sokol (1982) đã nói đến khả năng của cá nhân trong việc hoàn thành các hành vi khởi nghiệp. Mặt khác, khái niệm cảm nhận về sự tự tin khởi nghiệp khác với cảm nhận về khả năng kiểm soát hành vi ở dự cảm về việc có thể thực hiện được hành vi, là cảm nhận về việc có khả năng kiểm soát hành vi, thúc đẩy sự tự tin 
khởi nghiệp. Nhận thức kiểm soát hành vi đã được khẳng định có ảnh hưởng tích cực đến ý định khởi nghiệp của sinh viên trong các nghiên cứu của (Chau \& Huynh, 2020; D. T. T. Le \& Nguyen, 2016; T. N. D. Le \& Nguyen, 2019; T. A. Phan \& Tran, 2017).

\section{H4: nhận thức kiểm soát hành vi ảnh hưởng thuận chiều đến sụ tụ tin về tính khả thi khởi nghiệp}

\subsubsection{Chuẩn chủ quan}

Theo Liñán và Chen (2006), chuẩn chủ quan thể hiện sự phản đối hay ủng hộ của những người quan trọng nhất đối với một cá nhân (người thân, bạn bè) với việc khởi nghiệp kinh doanh; hay nhận thức về sự ảnh hưởng từ phía cộng đồng xã hội được định nghĩa là nhận thức về áp lực xã hội đến việc thực hiện hoặc không thực hiện hành vi (Ajzen, 1991). Đó là ảnh hưởng của những người quan trọng và gần gũi có thể tác động đến cá nhân thực hiện hành vi. Chuẩn chủ quan đã được khẳng định có ảnh hưởng tích cực đến ý định khởi nghiệp của sinh viên trong các nghiên cứu của (Chau \& Huynh, 2020; T. N. D. Le \& Nguyen, 2019; Ngo \& Cao, 2016; T. A. Phan \& Tran, 2017).

\section{H5: Chuẩn chủ quan ảnh hưởng thuận chiều đến sụ tụ tin về tính khả thi khởi nghiệp}

\subsubsection{Môi trưòng giáo dục}

Có nhiều nghiên cứu đã khẳng định môi trường giáo dục có ảnh hưởng tích cực đến ý định khởi nghiệp của sinh viên (Vojak, Griffin, Price, \& Perlov, 2006). Giáo dục sẽ cung cấp cho sinh viên những kiến thức, kỹ năng và thái độ để theo đuổi sự nghiệp kinh doanh (Ooi, Selvarajah, \& Meyer, 2011). Môi trường giáo dục được cho là đóng vai trò trong bồi dưỡng tinh thần kinh doanh cùng hoạt động trải nghiệm của sinh viên để tự tin để khởi nghiệp. Việc tham gia các chương trình đào tạo về khởi nghiệp đóng góp rất nhiều đến sự hình thành và phát triển ý định khởi nghiệp của sinh viên (Koe, 2016). DNC khuyến khích các hoạt động khởi nghiệp thông qua các chính sách có nhiệm vụ là tập trung thay đổi tư duy, hướng tới việc đưa sinh viên nghĩ về việc tạo ra giá trị hơn là tìm kiếm một việc làm. Các chính sách, chương trình đào tạo của nhà trường đều nhằm khuyến khích, là nền tảng, tạo cho sinh viên sự tự tin khởi nghiệp. Môi trường giáo dục đã được khẳng định có ảnh hưởng tích cực đến ý định khởi nghiệp của sinh viên trong các nghiên cứu của (Chau \& Huynh, 2020; T. N. D. Le \& Nguyen, 2019; Ngo \& Cao, 2016).

\section{H6: Môi trường giáo dục ảnh hưởng thuận chiều đến sụ tụ tin về tính khả thi khởi nghiệp}

\subsubsection{Sự tụ tin về tính khả thi}

Sự tự tin về tính khả thi là tin tưởng vào khả năng của bản thân, chủ động trong mọi công việc, dám tự quyết định và hành động một cách đúng đắn mà bản thân đó có thể bắt đầu công việc kinh doanh. Ý định khởi nghiệp sẽ xuất hiện khi cá nhân phát hiện ra một cơ hội mà họ thấy khả thi và mong muốn nắm lấy cơ hội đó. Cá nhân có mong muốn khởi nghiệp và tin tưởng vào năng lực khởi nghiệp thành công của bản thân sẽ xuất hiện tiềm năng khởi nghiệp. (Krueger \& Brazeal, 1994; Shapero \& Sokol, 1982). Lý thuyết về hành vi dự định của Ajzen (1991), cho rằng trước khi đi đến thực hiện một hành vi, con người phải có ý định về hành vi đó. Theo Matlay và cộng sự (2013), sự tự tin có tác động tích cực đến ý định khởi nghiệp; niềm tin vào sự thành công, tính hợp lý và sự phù hợp của ý định kinh doanh sẽ thúc đẩy ý tưởng quyết tâm thực hiện ý tưởng đó. Những cá nhân tự tin vào năng lực bản thân cao sẽ nỗ lực hết sức, nếu thực hiện tốt sẽ dẫn đến kết quả thành công. Chính vì vậy, khi có cơ hội kinh doanh thì tâm thế can đảm chấp nhận rủi ro tạo sự tự tin để bước vào khởi nghiệp là điều trước tiên cần có. Nghĩa là, sinh viên nhận thấy việc tự kinh doanh là rất dễ dàng, tự tin mình sẽ thành công khi khởi nghiệp hay cảm nhận rằng bản thân hoàn toàn có thể kiểm soát được hoạt động kinh doanh. Bên cạnh đó, sinh viên DNC mặc dù có ý tưởng kinh doanh, nhưng không đủ tự tin sẽ khó có thể bắt đầu khởi nghiệp kinh doanh dù được sự hỗ trợ từ phía nhà trường và gia đình. Các nghiên cứu cũng chỉ ra sự tự tin về tính khả thi tác động 
đến ý định khởi nghiệp (Chau \& Huynh, 2020; H. T. Nguyen \& Nguyen, 2016).

\section{H7: Sự tụ tin về tính khả thi ảnh hưởng thuận chiều đến ý định khởi nghiệp của sinh viên}

\subsubsection{Mô hình nghiên cứu đề xuất}

Dựa trên lược khảo các nghiên cứu trong và ngoài nước, kế thừa có chọn lọc các nhân tố ảnh hưởng đến ý định khởi nghiệp của sinh viên trong các nghiên cứu có liên quan (Chau \& Huynh, 2020; H. T. Nguyen \& Nguyen, 2016); đồng thời căn cứ vào các lý thuyết hành vi dự định (TPB), lý thuyết hành động hợp lý (TRA), lý thuyết sự kiện khởi nghiệp kinh doanh (SEE). Tuy nhiên, các nhà nghiên cứu vẫn có thể đưa thêm một số biến tác động vào mô hình nghiên cứu, việc đưa thêm các biến khả dĩ khác vào mô hình có thể đem lại tính chính xác cao hơn trong việc dự đoán ý định bởi xu hướng hành động không chỉ phụ thuộc duy nhất vào các yếu tố cảm nhận của cá nhân với hành vi khởi nghiệp hay ý kiến của những người xung quanh (Koe, 2016). Niềm tin vào sự thành công và sự phù hợp của của ý định kinh doanh sẽ thúc đẩy cá nhân quyết tâm thực hiện ý tưởng đó. Cá nhân sẽ tập trung mọi nguồn lực để thực hiện hành vi nhằm đạt được mục tiêu. Trong nghiên cứu này sử dụng mô hình cấu trúc tuyến tính (SEM) để kiểm định mô hình và các giả thuyết nghiên cứu. Mô hình nghiên cứu đề xuất gồm: (1) đặc điểm tính cách, (2) thái độ với hành vi khởi nghiệp, (3) nguồn vốn, (4) nhận thức kiểm soát hành vi, (5) chuẩn chủ quan và (6) môi trường giáo dục ảnh hưởng đến ý định khởi nghiệp qua biến trung gian sự tự tin về tính khả thi.

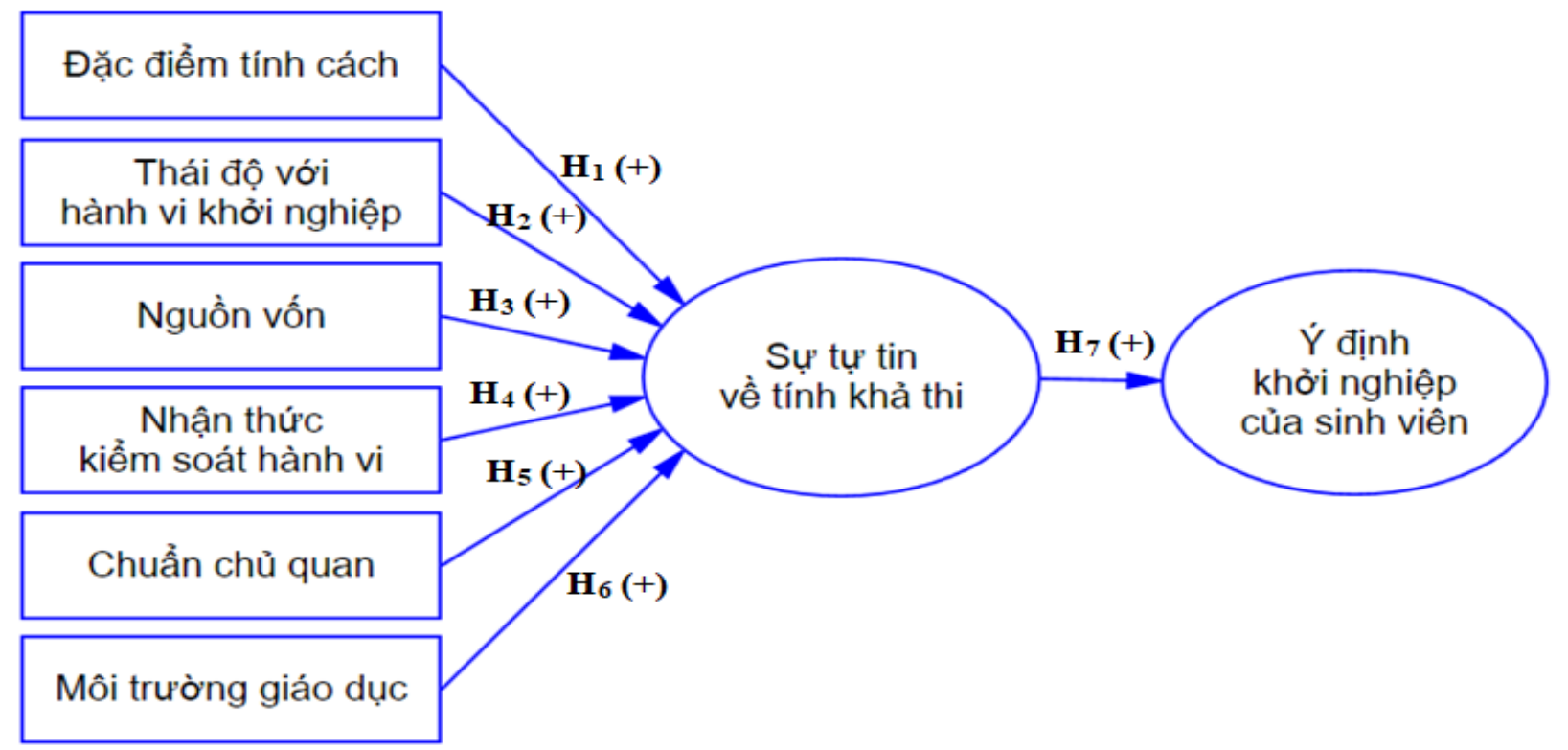

Hình 1. Mô hình nghiên cứu đề xuất

\section{Phương pháp nghiên cứu}

Kết hợp giữa phương pháp nghiên cứu định tính và định lượng: Nghiên cứu định tính được thực hiện qua thảo luận nhóm và lấy ý kiến chuyên gia (01 giảng viên giảng dạy về khởi nghiệp và 04 nhà quản lý doanh nghiệp có kinh nghiệm về khởi nghiệp) nhằm nhận diện những nhân tố tác động đến ý định khởi nghiệp của của sinh viên; xác định mô hình nghiên cứu và hiệu chỉnh thang đo sơ bộ phù hợp với địa bàn nghiên cứu (thang đo trong nghiên cứu kế thừa những thang đo đã được kiểm định qua các nghiên cứu trước). Nhóm đã thực hiện thảo luận để xác định các nhân tố nào ảnh hưởng đến ý định khởi nghiệp của của sinh viên; xây dựng mô hình nghiên cứu và hiệu chỉnh thang đo. Tiếp đến, phỏng vấn nhanh 30 sinh viên đang có ý định khởi nghiệp tại trường DNC theo phương pháp chọn mẫu thuận tiện để đánh giá bảng câu hỏi sơ bộ, từ đó hiệu 
chỉnh các thang đo cho phù hợp thực tiễn và kiểm định đạt độ tin cậy để hình thành kết quả là bảng câu hỏi chính thức. Nghiên cứu định lượng: sử dụng thang đo Likert 5 mức độ, để lượng hóa mối quan hệ giữa các nhân tố thông qua các phần mềm thống kê, được thực hiện qua các bước kiểm định độ tin cậy Cronbach's Alpha, phân tích nhân tố khám phá (EFA), phân tích (CFA) và phân tích mô hình cấu trúc tuyến tính (SEM) để kiểm định mô hình và các giả thuyết nghiên cứu.

Theo Hair, Anderson, Tatham, và Black (1998), cho rằng kích thước mẫu cần tối thiểu phù hợp đối với phân tích nhân tố khám phá (EFA) là một biến cần có 05 quan sát. Nghiên cứu có 06 thang đo với 26 biến quan sát, vì vậy cỡ mẫu tối thiểu là: $26 * 5=130$ quan sát; cỡ mẫu với các quan điểm tương ứng: $100=$ tệ; $200=$ khá; $300=$ tốt; 500 = rất tốt; 1000 hoặc hơn = tuyệt vời (Comrey \& Lee, 1992). Trong nghiên cứu này xác định cỡ mẫu là 310, theo Comrey và Lee (1992) cỡ mẫu xác định là đạt mức tốt. Phương pháp thu thập dữ liệu là khảo sát trực tiếp sinh viên dựa vào bảng câu hỏi soạn sẵn (do hạn chế về thời gian và chi phí nghiên cứu) nên cơ cấu cỡ mẫu được phân bổ tại 02 Khoa của DNC.

Nghiên cứu sử dụng phương pháp chọn mẫu xác suất phân tầng, cơ cấu phân bổ cỡ mẫu theo số lượng sinh viên năm thứ ba và năm thứ tư (lý do lựa chọn sinh viên hai năm cuối bởi vì đây là giai đoạn sinh viên chú ý nhiều hơn tới vấn đề định hướng nghề nghiệp sau khi tốt nghiệp). Theo T. D. Nguyen (2011), phương pháp phân tích phân tầng chia tổng thể ra thành nhiều nhóm nhỏ, được thực hiện theo tỷ lệ hoặc không theo tỷ lệ. Khung mẫu được xác định là đối tượng sinh viên năm thứ ba và tư của 02 Khoa với tỷ lệ tương ứng được phân theo tỷ lệ sinh viên của các ngành: Khoa Kiến trúc - Xây dựng và môi trường (với 04 ngành Kỹ thuật xây dựng; Kiến trúc; Quản lý đất đai; Quản lý tài nguyên và Môi trường) và Khoa Kinh tế (với 02 lớp thuộc ngành Quản trị kinh doanh). Thực hiện lấy mẫu theo tỷ lệ (\%) số sinh viên của các ngành. Căn cứ theo danh sách của lớp học, dùng hàm random () trong Excel lấy ngẫu nhiên số sinh viên để chọn đủ số lượng. Sau đó tiến hành khảo sát theo danh sách sinh viên ngẫu nhiên được chọn, bằng bảng câu hỏi soạn sã̃n. Dũ liệu sau khi thu thập từ các đối tượng khảo sát được mã hóa, làm sạch, phân tích qua các bước: đánh giá độ tin cậy của thang đo thông qua hệ số Cronbach's Alpha, phân tích nhân tố khám phá (EFA), phân tích nhân tố khẳng định (CFA), phân tích mô hình cấu trúc tuyến tính (SEM) để kiểm định mô hình và các giả thuyết nghiên cứu.

\section{Kết quả nghiên cứu}

\subsection{Thống kê mô tả về mẫu nghiên cứu}

Với 320 phiếu khảo sát được thu về và làm sạch dữ liệu đạt được 310 phiếu hợp lệ, kết quả phân tích dữ liệu khảo sát (Bảng 1) về giới tính, năm học, ngành học như sau:

\section{Bảng 1}

Kết quả thống kê dữ liệu

\begin{tabular}{ccccccccc}
\hline \multicolumn{1}{c}{ Giới tính } & \multicolumn{1}{c}{ Năm học } & \multicolumn{6}{c}{ Ngành học } \\
\hline $\begin{array}{c}\text { Nam } \\
(\%)\end{array}$ & $\begin{array}{c}\text { Nũ } \\
(\%)\end{array}$ & $\begin{array}{c}3 \\
(\%)\end{array}$ & $\begin{array}{c}4 \\
(\%)\end{array}$ & $\begin{array}{c}\text { Kiến trúc } \\
(\%)\end{array}$ & $\begin{array}{c}\text { Kỹ thuật } \\
\text { xây dựng } \\
(\%)\end{array}$ & $\begin{array}{c}\text { Quản lý } \\
\text { đất đai } \\
(\%)\end{array}$ & $\begin{array}{c}\text { Quản lý tài } \\
\text { nguyên và } \\
\text { Môi trường } \\
(\%)\end{array}$ & $\begin{array}{c}\text { Quản trị } \\
\text { kinh doanh } \\
(\%)\end{array}$ \\
\hline 73.5 & 26.5 & 42.0 & 58.0 & 19.0 & 37.0 & 10.0 & 8.0 & 26.0 \\
\hline
\end{tabular}




\subsection{Kiểm định độ tin cậy của thang đo}

Các thang đo đạt độ tin cậy vì có kết quả hệ số Cronbach's Alpha > 0.6; hệ số tương quan biến tổng đều lớn hơn 0.5 . Như vậy, thang đo phù hợp sử dụng cho bước phân tích nhân tố khám phá (Hoang \& Chu, 2008).

\section{Bảng 2}

Kiểm định độ tin cậy thang đo

\begin{tabular}{|c|c|c|c|c|}
\hline Thang đo & $\begin{array}{c}\text { Tương } \\
\text { quan } \\
\text { biến tổng }\end{array}$ & $\begin{array}{c}\text { Hệ số } \\
\text { Cronbach' } \\
\text { Alpha nếu } \\
\text { loại biến }\end{array}$ & $\begin{array}{c}\text { Giá trị } \\
\text { trung } \\
\text { bình }\end{array}$ & $\begin{array}{c}\text { Nguồn } \\
\text { tham khảo }\end{array}$ \\
\hline
\end{tabular}

\section{1. Đặc điểm tính cách (TC): Cronbach’ Alpha = 0.891}

Tôi muốn được mọi người tôn trọng và biết đến

Tôi luôn thích trải nghiệm những cái mới

\begin{tabular}{|c|c|c|c|}
\hline 0.740 & 0.867 & 3.67 & (Ngo \& \\
\hline 0.786 & 0.849 & 3.65 & $\begin{array}{l}\text { Cao, 2016; } \\
\text { T. A. Phan }\end{array}$ \\
\hline 0.785 & 0.851 & 3.70 & \& Tran, \\
\hline 0.738 & 0.870 & 3.65 & 2017) \\
\hline
\end{tabular}

Tôi sẵn sàng chấp nhận rủi ro trong kinh doanh

Tôi luôn cố gắng để làm tốt hơn những người khác

\section{Thái độ đối với hành vi khởi nghiệp (TD): Cronbach’ Alpha $=0.890$}

Khởi nghiệp kinh doanh rất có sức hút với bạn

0.736

0.865

3.82

(T. A. Phan

Nếu bạn có cơ hội và nguồn lực, bạn thích khởi

0.692

0.875

3.79

nghiệp kinh doanh

Bạn cảm thấy rất hài lòng khi trở thành một chủ doanh nghiệp

$\begin{array}{llll}0.753 & 0.861 & 3.76 & \text { Thac, 2020) }\end{array}$

Trở thành một doanh nhân sẽ đem lại nhiều lợi ích hơn là bất lợi

$\begin{array}{lll}0.730 & 0.866 & 3.71\end{array}$

Khởi nghiệp kinh doanh có nhiều cơ hội để phát

0.748

0.862

3.84 triển bản thân

\section{Nguồn vốn $(\mathrm{NV})$ : Cronbach’ Alpha $=0.889$}

Có thể huy động vốn từ gia đình, người thân và

0.865

0.827

3.54

(L. K. Le, bạn bè để khởi nghiệp 2018;

Có khả năng tích lũy vốn để kinh doanh

0.855

0.838

3.54

Có thể huy động vốn từ những nguồn vốn khác

0.529

0.886

3.58

Truong \&

Nguyen,

Có thể vay vốn từ các gói vay dành riêng cho sinh

0.573

0.872

3.53 viên khởi nghiệp

\section{Nhận thức kiểm soát hành vi $(\mathrm{NT})$ : Cronbach' Alpha $=0.922$}

\begin{tabular}{|c|c|c|c|c|}
\hline $\begin{array}{l}\text { Bạn có thể kiểm soát quá trình bắt đầu một doanh } \\
\text { nghiệp mới }\end{array}$ & 0.665 & 0.905 & 4.00 & $\begin{array}{l}\text { (Chau \& } \\
\text { Huynh, }\end{array}$ \\
\hline $\begin{array}{l}\text { Bạn biết những chi tiết thực tế cần thiết để bắt đầu } \\
\text { một doanh nghiệp mới }\end{array}$ & 0.747 & 0.887 & 3.97 & $\begin{array}{l}\text { 2020; H. T. } \\
\text { Nguyen \& }\end{array}$ \\
\hline Bạn biết cách để phát triển một doanh nghiệp & 0.709 & 0.897 & 3.95 & \\
\hline
\end{tabular}




\begin{tabular}{|c|c|c|c|c|}
\hline Thang đo & $\begin{array}{l}\text { Tương } \\
\text { quan } \\
\text { biến tổng }\end{array}$ & $\begin{array}{c}\text { Hệ số } \\
\text { Cronbach' } \\
\text { Alpha nếu } \\
\text { loại biến }\end{array}$ & $\begin{array}{l}\text { Giá trị } \\
\text { trung } \\
\text { bình }\end{array}$ & $\begin{array}{c}\text { Nguồn } \\
\text { tham khảo }\end{array}$ \\
\hline $\begin{array}{l}\text { Hoàn toàn có thể kiểm soát được hoạt động kinh } \\
\text { doanh của mình khi khởi nghiệp }\end{array}$ & 0.649 & 0.905 & 4.02 & 2016) \\
\hline \multicolumn{5}{|l|}{ 5. Chuẩn chủ quan (CQ): Cronbach' Alpha = 0.899} \\
\hline Tin rằng nếu tự kinh doanh thì bạn bè sẽ ủng hộ & 0.607 & 0.870 & 3.70 & \multirow{4}{*}{$\begin{array}{c}\text { (Chau \& } \\
\text { Huynh, } \\
\text { 2020; T. A. } \\
\text { Phan \& } \\
\text { Tran, 2017) }\end{array}$} \\
\hline $\begin{array}{l}\text { Gia đình chắc chắn ủng hộ quyết định tự kinh } \\
\text { doanh của tôi }\end{array}$ & 0.654 & 0.858 & 3.67 & \\
\hline $\begin{array}{l}\text { Những người quan trọng sẽ ủng hộ quyết định tự } \\
\text { tạo dựng một doanh nghiệp }\end{array}$ & 0.611 & 0.869 & 3.72 & \\
\hline $\begin{array}{l}\text { Nếu gặp khó khăn trong việc kinh doanh thì sẽ } \\
\text { nhận được sự hỗ trợ của ngưởi thân và bạn bè }\end{array}$ & 0.549 & 0.883 & 3.64 & \\
\hline
\end{tabular}

\section{Môi trường giáo dục (GD): Cronbach’ Alpha = 0.884}

Giáo dục trong trường cung cấp những kỹ năng $\quad 0.507$

và năng lực cần thiết để bạn khởi nghiệp

Nhà trường khuyến khích phát triển ý tưởng sáng tạo để khởi nghiệp

Giáo dục trong trường cung cấp những kiến thức cần thiết về kinh doanh để bạn khởi nghiệp

Giáo dục trong trường khuyến khích sinh viên tham gia các hoạt động ngoại khóa về khởi nghiệp

\begin{tabular}{|c|c|c|c|}
\hline 0.507 & 0.868 & 3.67 & (Chau \& \\
\hline 0.585 & 0.844 & 3.67 & $\begin{array}{c}\text { 2020; Ngo } \\
\text { \& Cao, } \\
2016)\end{array}$ \\
\hline 0.612 & 0.838 & 3.80 & \\
\hline 0.574 & 0.852 & 3.76 & \\
\hline
\end{tabular}

\section{Sự tự tin về tính khả thi (KT): Cronbach' Alpha = 0.878} khó với tôi

Tin rằng hoàn toàn có thể tự kinh doanh trong

$\begin{array}{lll}0.561 & 0.839 & 3.58\end{array}$
tương lai

Tin rằng hoàn toàn có thể bắt đầu một doanh nghiệp

$0.843 \quad 3.60 \quad$ Nguyen,

Mục tiêu công việc là trở thành doanh nhân

0.555

0.840

3.56

\section{8. Ý định khởi nghiệp của sinh viên (YD): Cronbach’ Alpha =0.878}

\begin{tabular}{|c|c|c|c|c|}
\hline Muốn được tự làm chủ doanh nghiệp & 0.593 & 0.839 & 3.74 & (H. T. \\
\hline $\begin{array}{l}\text { Quyết tâm tạo ra một doanh nghiệp trong tương } \\
\text { lai }\end{array}$ & 0.610 & 0.833 & 3.74 & $\begin{array}{l}\text { Nguyen \& } \\
\text { Nguyen, } \\
2016 \cdot \text { V O }\end{array}$ \\
\hline Cố gắng hết sức để bắt đầu công việc kinh doanh & 0.505 & 0.859 & 3.59 & Phan \& \\
\hline 3ắt đầu khởi nghiệp khi còn đang học tại trường & 0.527 & 0.851 & 3.71 & Trac, 2020) \\
\hline
\end{tabular}




\subsection{Phân tích nhân tố khám phá (EFA)}

Kết quả phân tích nhân tố khám phá (EFA) bằng phương pháp xoay (Promax) cho thấy, 33 biến quan sát đều đạt yêu cầu về giá trị cụ thể: Hệ số $\mathrm{KMO}=0.865$ nằm trong khoảng từ $[0.5$; $1.0]$ thỏa mãn điều kiện; kiểm định Bartlett với hệ số (sig.) $=0.00<0.05$; tổng phương sai trích là $68.101 \%$ ( $>50 \%$ ) đạt yêu cầu cho biết các nhân tố có ý nghĩa thống kê; thỏa điều kiện với Eigenvalue $=1.454>1$ (Hoang \& Chu, 2008); hệ số tải các nhân tố đều $>0.5$ chứng tỏ các biến quan sát này có độ tin cậy (Hair, Black, Babin, Anderson, \& Tatham, 2006). Kết luận phân tích nhân tố (EFA) phù hợp để phân tích nhân tố khẳng định (CFA).

\subsection{Kết quả phân tích nhân tố khẳng định (CFA)}

Kết quả phân tích các thành phần của thang đo (Hình 2) đều đạt được giá trị hội tụ, giá trị phân biệt, tính đơn hướng, độ tin cậy:

Kết quả phân tích có 467 bậc tự do; giá trị $\mathrm{df}=467 ; \mathrm{Chi}^{2}=752.951$; Chi-Square/df $=1.612$ $<3$; chỉ số TLI $=0.951>0.9$; CFI $=0.957>0.9$; GFI $=0.876>0.8$ là đạt (Taylor, Sharland, Cronin, \& Bullard, 1993); RMSEA = $0.045<0.06$ mô hình đạt độ tương thích với dữ liệu thị trường (Hooper, Coughlan, \& Mullen, 2008). Các trọng số đã chuẩn hóa từ $(0.618-0.969)$ đều $>$ 0.5 thang đo đạt giá trị hội tụ; không có sự tương quan giữa các sai số của các biến quan sát nên đều đạt tính đơn hướng (Anderson \& Gerbing, 1988). Các giá trị $\mathrm{P}$-value đều $<0.05$ nên hệ số tương quan của từng cặp khái niệm khác biệt so với 1 ở độ tin cậy $95 \%$ đạt giá trị phân biệt (Steenkamp \& Van Trijp, 1991). Kết quả độ tin cậy tổng hợp $\left(\mathrm{P}_{\mathrm{c}}\right)$ của các thang đo đều lớn $>0.5$ và phương sai trích $\left(\mathrm{P}_{\mathrm{vc}}\right)$ của các thang đo đều lớn $>0.5$. Do đó các thang đo trong mô hình nghiên cứu đều đạt độ tin cậy. Kết luận, mô hình nghiên cứu phù hợp để tiếp tục phân tích cấu trúc tuyến tính SEM (Taylor et al., 1993).

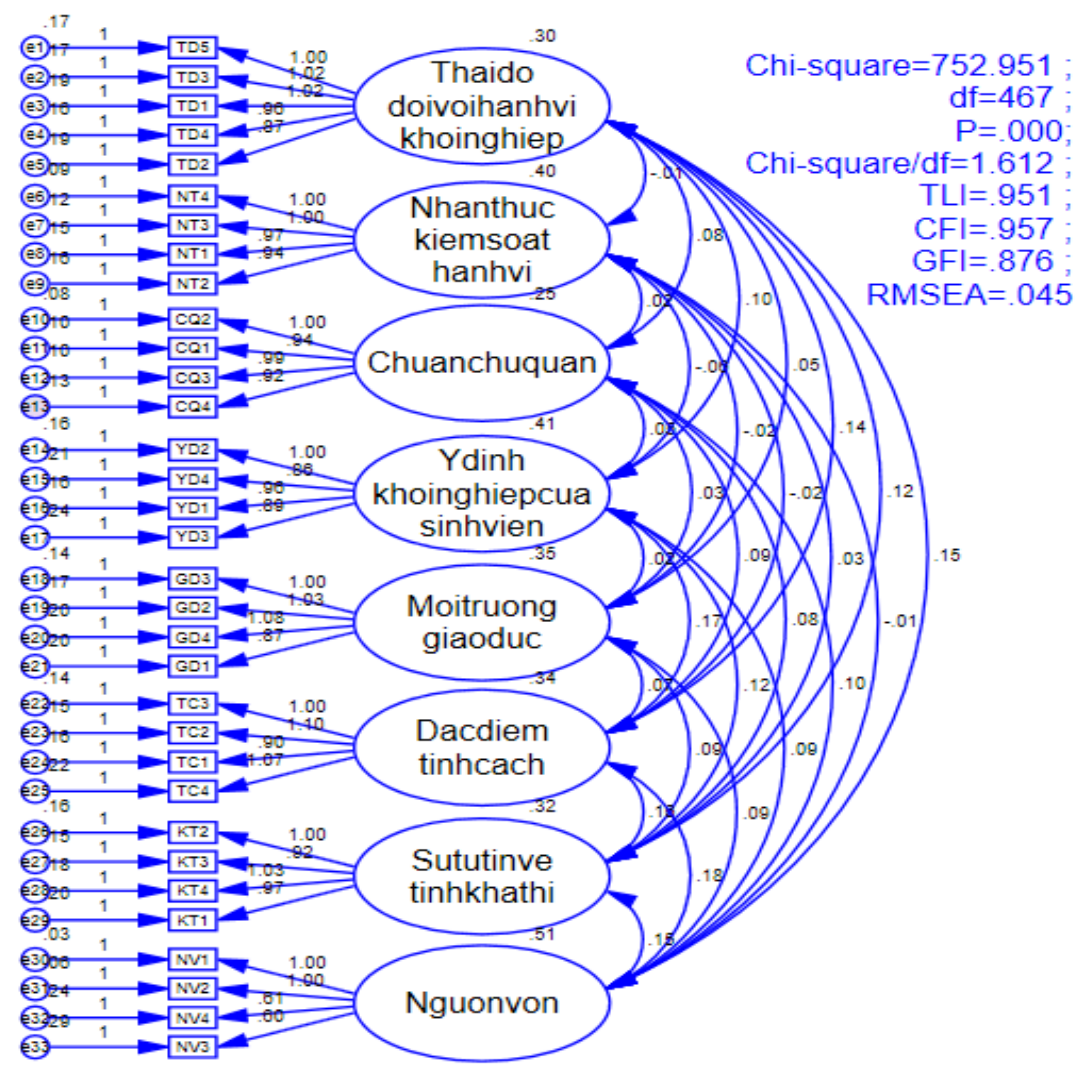

Hình 2. Mô hình tới hạn đo lường các khái niệm trong mô hình (chuẩn hóa) 


\subsection{Kết quả phân tích mô hình cấu trúc tuyến tính (SEM)}

Kết quả phân tích mô hình SEM cho thấy mô hình có 361 bậc tự do; các chỉ số (Hình 3) giá trị $\mathrm{Chi}^{2}=795.926 ; \mathrm{df}=473 ;$ Chi-Square $/ \mathrm{df}=1.683<3$; chỉ số $\mathrm{TLI}=0.945>0.90 ; \mathrm{CFI}=$ $0.951>0.9 ; \mathrm{GFI}=0.869>0.8$ là đạt; RMSEA $=0.047<0.06$ là tốt (Hooper et al., 2008).

Các giả thuyết nghiên cứu sẽ được kiểm định bằng cách xem xét mối quan hệ nhân quả giữa các khái niệm nghiên cứu, kết quả (Bảng 3 ) cho thấy:

- Sự tương quan giữa nhân tố đặc điểm tính cách và sự tự tin về tính khả thi có $\mathrm{P}=0.000$ $<0.01$; hệ số chuẩn hóa $(\beta=0.329)$, khẳng định giả thuyết $\mathrm{H} 1$ được chấp nhận, cho thấy đặc điểm tính cách có ảnh hưởng thuận chiều dương đến sự tự tin về tính khả thi ở mức ý nghĩa $1 \%$ (độ tin cậy $99 \%$ );

- Sự tương quan giữa nhân tố thái độ đối với hành vi khởi nghiệp và sự tự tin về tính khả thi có $\mathrm{P}=0.016<0.05$; hệ số chuẩn hóa $(\beta=0.162)$, khẳng định giả thuyết $\mathrm{H} 2$ được chấp nhận, cho thấy thái độ đối với hành vi khởi nghiệp có ảnh hưởng thuận chiều dương đến sự tự tin về tính khả thi ở mức ý nghĩa 5\% (độ tin cậy 95\%);

- Sự tương quan giữa nhân tố nguồn vốn và sự tự tin về tính khả thi có $\mathrm{P}=0.046<0.05$; hệ số chuẩn hóa $(\beta=0.099)$, khẳng định giả thuyết $\mathrm{H} 3$ được chấp nhận, cho thấy nguồn vốn có ảnh hưởng thuận chiều dương đến sự tự tin về tính khả thi ở mức ý nghĩa $5 \%$ (độ tin cậy $95 \%$ );

- Sự tương quan giữa nhân tố nhận thức kiểm soát hành vi và sự tự tin về tính khả thi có $\mathrm{P}$ $=0.036<0.05$; hệ số chuẩn hóa $(\beta=0.101)$, khẳng định giả thuyết $\mathrm{H} 4$ được chấp nhận, cho thấy nhận thức kiểm soát hành vi có tác động thuận chiều dương đến sự tự tin về tính khả thi ở mức ý nghĩa 5\% (độ tin cậy 95\%);

- Nhân tố chuẩn chủ quan có hệ số $\mathrm{P}=0.165>0.05$ không có ý nghĩa thống kê tại mức ý nghĩa $5 \%$ (độ tin cậy $95 \%$ ), nên có thể kết luận chuẩn chủ quan không có ý nghĩa thống kê, nghĩa là không tìm thấy mối liên hệ ảnh hưởng tuyến tính ý kiến của bạn bè, gia đình, tác động đến ý định khởi nghiệp của sinh viên, bác bỏ giả thuyết (H5). Kết quả này chưa phù hợp với các kết quả nghiên cứu trước của (Chau \& Huynh, 2020; Ngo \& Cao, 2016; T. A. Phan \& Tran, 2017) nhưng lại phù hợp với nghiên cứu của (Boissin, Branchet, Emin, \& Herbert, 2009; Liñán \& Chen, 2006). Điều này có thể lý giải là: dù ý kiến của gia đình, bạn bè hay người thân có ủng hộ việc kinh doanh của sinh viên hay phản đối thì bản thân sinh viên cũng quyết tâm kinh doanh vì các em tin vào khả năng bản thân mình làm được, chấp nhận rủi ro để làm, tuy nhiên nếu được gia đình ủng hộ thì sẽ tốt hơn, tinh thần mình thoải mái hơn và sinh viên sẽ có thêm động lực để kinh doanh;

- Sự tương quan giữa nhân tố môi trường giáo dục và sự tự tin về tính khả thi có $\mathrm{P}=0.011$ $<0.05$; hệ số chuẩn hóa $(\beta=0.139)$, khẳng định giả thuyết H6 được chấp nhận, cho thấy môi trường giáo dục có ảnh hưởng thuận chiều dương đến sự tự tin về tính khả thi ở mức ý nghĩa 5\% (độ tin cậy 95\%);

- Sự tương quan giữa sự tự tin về tính khả thi và ý định khởi nghiệp của sinh viên có $\mathrm{P}=$ $0.000<0.01$; hệ số chuẩn hóa $(\beta=0.385)$ cho thấy nhân tố sự tự tin về tính khả thi có tác động đến ý định khởi nghiệp của sinh viên ở mức ý nghĩa $1 \%$ (độ tin cậy $99 \%$ ); và cứ 01 đơn vị sự tự tin về tính khả thi thì có tác động đến 0.385 đơn vị ý định khởi nghiệp của sinh viên. 


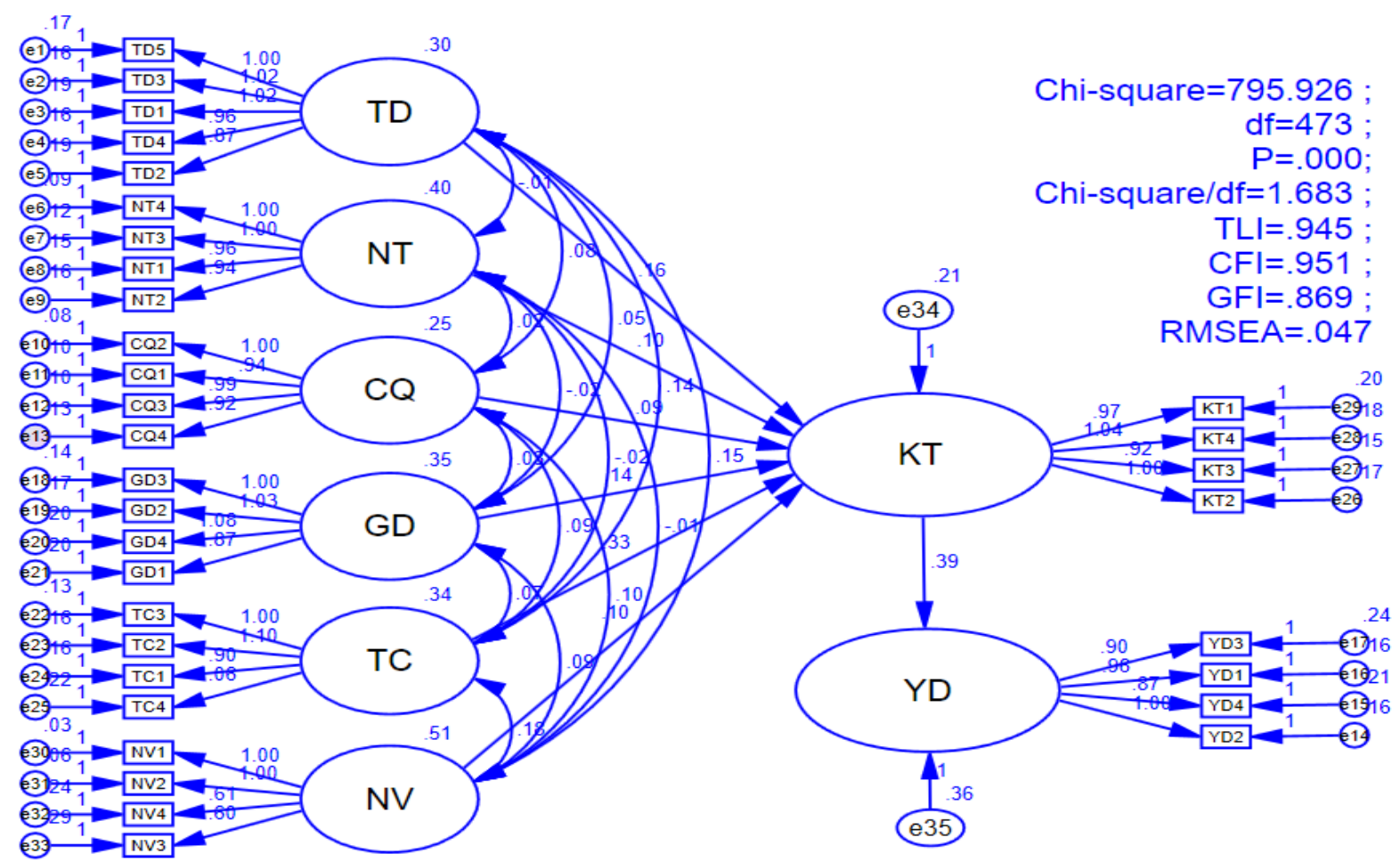

Hình 3. Kết quả kiểm định mô hình cấu trúc (SEM) tới hạn

Nguồn: Kết quả phân tích dữ liệu khảo sát 310 sinh viên DNC (2020)

\section{Bảng 3}

Kiểm định quan hệ giữa các khái niệm trong mô hình (chưa chuẩn hóa)

\begin{tabular}{cccccccc}
\hline \multicolumn{2}{c}{ Tác động } & & Ước lượng & S.E. & C.R. & P & Giả thuyết \\
\hline KT & $<---$ & TD & 0.162 & 0.067 & 2.415 & 0.016 & Chấp nhận \\
KT & $<---$ & NT & 0.101 & 0.048 & 2.096 & 0.036 & Chấp nhận \\
KT & $<---$ & CQ & 0.094 & 0.068 & 1.387 & 0.165 & Bác bỏ \\
KT & $<---$ & GD & 0.139 & 0.055 & 2.550 & 0.011 & Chấp nhận \\
KT & $<---$ & TC & 0.329 & 0.067 & 4.885 & $* * *$ & Chấp nhận \\
KT & $<---$ & NV & 0.099 & 0.050 & 1.999 & 0.046 & Chấp nhận \\
YD & $<---$ & KT & 0.385 & 0.072 & 5.356 & $* * *$ & Chấp nhận \\
\hline
\end{tabular}

Nguồn: Kết quả phân tích dữ liệu khảo sát 310 sinh viên DNC (2020)

Kết quả kiểm định Bootstrap được xem là phương pháp lấy mẫu lặp lại có thay thế, trong đó mẫu ban đầu đóng vai trò là đám đông (Schumacker \& Lomax, 2006). Trong nghiên cứu này, nhóm tác giả đã cho lặp lại cỡ mẫu 500 khác bằng phương pháp Bootstrap nhằm kiểm định tính ổn định của các ước lượng. Từ dữ liệu phân tích trong bảng (Bảng 4$)$ cho thấy, các giá trị $|\mathrm{CR}|$ đều < 1.96; suy ra P > 5\%, độ lệch là rất nhỏ, không có ý nghĩa thống kê ở độ tin cậy $95 \%$. Kết luận mô hình ước lượng có thể tin cậy được và đây cũng là kết quả mong đợi khi phân tích mô hình cấu trúc tuyến tính $(\mathrm{SEM})$. Các nhân tố có ảnh hưởng đến ý định khởi nghiệp của sinh viên là: đặc điểm tính cách; thái độ đối với hành vi khởi nghiệp; môi trường giáo dục; nhận thức kiểm soát hành vi và nguồn vốn. 


\section{Bảng 4}

Kết quả kiểm định Boostrap

\begin{tabular}{ccccccccc}
\hline \multicolumn{2}{c}{ Tác động } & SE & SE-SE & Mean & Bias & SE-Bias & CR \\
\hline KT & $<---$ & TD & 0.072 & 0.002 & 0.165 & 0.003 & 0.003 & 1.00 \\
KT & $<---$ & NT & 0.047 & 0.001 & 0.106 & 0.003 & 0.002 & 1.50 \\
KT & $<---$ & CQ & 0.071 & 0.002 & 0.088 & -0.006 & 0.003 & -2.00 \\
KT & $<---$ & GD & 0.057 & 0.002 & 0.140 & 0.001 & 0.003 & 0.33 \\
KT & $<---$ & TC & 0.073 & 0.002 & 0.328 & -0.001 & 0.003 & -0.33 \\
KT & $<---$ & NV & 0.055 & 0.002 & 0.105 & 0.003 & 0.002 & 1.50 \\
YD & $<---$ & KT & 0.080 & 0.003 & 0.382 & -0.003 & 0.004 & -0.75 \\
\hline
\end{tabular}

Nguồn: Kết quả phân tích dữ liệu khảo sát 310 sinh viên DNC (2020)

\section{Kết luận và hàm ý quản trị}

Nghiên cứu đã xác định được các nhân tố và mức độ tác động của từng nhân tố đến ý định khởi nghiệp của sinh viên. Vì vậy, để nâng cao ý định khởi nghiệp của sinh viên Trường Đại học Nam Cần Thơ, nhóm tác giả đề xuất một số hàm ý quản trị được ưu tiên thực hiện như sau:

Đặc điểm tính cách: là nhân tố có mức ảnh hưởng quan trọng nhất đến ý định khởi nghiệp của sinh viên, vì vậy việc tự trang bị cho bản thân nâng cao kiến thức và kỹ năng cần thiết về khởi nghiệp kinh doanh, bên cạnh nền tảng kiến thức khoa học công nghệ, tăng cường tính tự học, tìm hiểu về những mô hình kinh doanh thành công sẽ giúp tăng năng lực cảm nhận ở sinh viên để nâng cao ý định khởi nghiệp. Các hoạt động ngoại khóa liên quan đến khởi nghiệp và kinh doanh cần được Nhà trường xây dựng trong chương trình đào tạo, đấy sẽ là nền tảng để sinh viên phát triển kỹ năng và gia tăng ý định khởi nghiệp. Người thực hiện khởi nghiệp rất cần những yếu tố sáng tạo, nhạy bén để có thể cải tiến về mẫu mã, tính năng, chất lượng, giá cả và ngay cả cách thức marketing để đưa sản phẩm, dịch vụ tới người tiêu dùng mới có thể hy vọng khởi nghiệp thành công. Đồng thời, tạo cơ hội cho sinh viên rèn luyện những tố chất cần thiết đối với một nhà quản trị như: bản lĩnh, tự tin, năng động, sáng tạo, có nghệ thuật tổ chức, quản lý, điều hành. Qua đó, năng lực lãnh đạo được vun đắp, là động lực để phát triển ý định khởi nghiệp. Nhà trường cần tạo điều kiện để sinh viên tích cực tham gia các hoạt động nghiên cứu khoa học, tích góp kinh nghiệm kinh doanh ngoài xã hội nếu có điều kiện. Muốn khởi nghiệp thuận lợi, sinh viên cần nắm vững chuyên môn sâu thuộc lĩnh vực khởi nghiệp, vì vậy nhà trường sinh viên cần nâng cao tinh thần tự học của sinh viên, giúp sinh viên học tập chăm chỉ, tiếp thu tốt kiến thức chuyên môn, tranh thủ tìm hiểu thêm kiến thức thực tế để đáp ứng yêu cầu khởi nghiệp.

Thái độ với hành vi khởi nghiệp: là nhân tố có mức tác động mạnh thứ hai đến ý định khởi nghiệp của sinh viên, vì vậy những kiến thức, kỹ năng được học kết hợp với kiến thức khởi nghiệp và thái độ tích cực với việc thành lập doanh nghiệp sẽ là hành trang trên hành trình nâng cao ngọn lửa khởi nghiệp của sinh viên. Nhà trường cần thiết lập các nhóm, các Trung tâm hỗ trợ khởi nghiệp tại trường, tại các Khoa chuyên môn, nhằm nâng cao thái độ của từng sinh viên, từng nhóm sinh viên đối với các ý tưởng khởi nghiệp. Để tạo nên hứng thú về nghề nghiệp, "tư duy làm chủ thay vì làm thuê" luôn là phương châm để sinh viên muốn thay đổi tương lai. Do đó, để khơi dậy thái độ với hành vi khởi nghiệp, nhà trường cần tăng cường giới thiệu các tấm gương đã khởi nghiệp thành công, các mô hình khởi nghiệp tiêu biểu của Việt Nam cũng như thế giới để khơi dậy ham muốn kinh doanh. Giúp sinh viên chủ động và tích cực tham gia các hoạt động trải nghiệm 
thực tiễn kinh doanh do nhà trường, các câu lạc bộ, các tổ chức đoàn thể và các hội thảo về khởi nghiệp dành cho sinh viên. Qua đó giúp sinh viên làm chủ, tự quản lý bản thân, đề ra những ý tưởng kinh doanh khi còn ngồi trên ghế nhà trường, mở rộng mối quan hệ, học hỏi kinh nghiệm từ những người bạn, người thân thành lập doanh nghiệp, để được chia sẻ kinh nghiệm từ những người có nhiều kinh nghiệm thực tế, lường trước những khó khăn, thử thách khi kinh doanh.

Môi trường giáo dục: là nhân tố có mức ảnh hưởng mạnh thứ ba đến ý định khởi nghiệp của sinh viên, được xem như là nhân tố quan trọng hình thành nên tư duy lập nghiệp và khơi dậy lòng ham muốn kinh doanh ngay khi còn ngồi trên ghế nhà trường. Chính vì thế, để tặng sự đam mê của sinh viên đối với khởi nghiệp kinh doanh, nhà trường nên bổ sung các học phần cơ bản liên quan đến khởi nghiệp để gia tăng các kiến thức và kỹ năng, kinh nghiệm thiết yếu khi bắt đầu khởi nghiệp, giúp sinh viên gia tăng ý định khởi nghiệp. Nhà trường cần có định hướng đối với các giảng viên trong quá trình giảng dạy khuyến khích sinh viên khởi nghiệp; thành lập vườn ươm doanh nghiệp; tạo mối liên kết chặt chẽ với cộng đồng doanh nhân và doanh nghiệp, kết nối với sinh viên thông qua các hoạt động tham quan thực tế doanh nghiệp, tiếp thu kinh nghiệm, thực tập và làm việc sau khi tốt nghiệp. Tăng cường các hoạt động truyền thông về các cuộc thi khởi nghiệp, kết nối doanh nghiệp để tổ chức ngày hội việc làm. Thường xuyên tổ chức các ngày hội kinh doanh, hội chợ; tổ chức các cuộc thi định hướng khởi nghiệp như ý tưởng kinh doanh, hội thảo về khởi nghiệp, mô phỏng lên kế hoạch kinh doanh, xây dựng mạng lưới cựu sinh viên khởi nghiệp. Thành lập các câu lạc bộ khởi nghiệp để tăng cường giới thiệu, phổ biến hình mẫu trong cộng đồng sinh viên, chủ doanh nghiệp thành đạt, các mô hình khởi nghiệp và kinh nghiệm làm giàu của các doanh nhân trẻ.

Kiểm soát nhận thức hành vi: là nhân tố có mức ảnh hưởng thứ tư đến ý định khởi nghiệp, nó phản ánh sự tự tin của sinh viên khi quyết định khởi nghiệp. Ngoài đam mê, khát vọng và động lực là điều kiện cần song chưa đủ để khởi nghiệp kinh doanh. Kỷ luật và quyết tâm là những yếu tố đảm bảo để các doanh nhân đi theo và phát triển các ý tưởng kinh doanh, bất luận quá trình này suôn sẻ hay phải đối mặt với nhiều trở ngại, sinh viên cần có một suy nghĩ độc lập, hình thành ý thức "dám nghĩ, dám làm", xem việc khởi sự doanh nghiệp như một trải nghiệm kiến thức thực tế trong môi trường xã hội góp phần hình thành nên kinh nghiệm và thành công trong hoạt động nghề nghiệp. Để tạo cho sinh viên có cơ hội "thực học, thực nghiệm" thì nhà trường đóng vai trò rất quan trọng giúp sinh viên trau dồi kinh nghiệm, nâng cao nhận thức ngay từ khi còn học. Từ đó, nâng cao sự cảm nhận của cá nhân, tạo sự tự tin để làm một chủ doanh nghiệp trong tương lai.

Nguồn vốn: là nhân tố ít quan trọng nhất ảnh hưởng đến ý định khởi nghiệp của sinh viên DNC. Bởi vì, công ty khởi nghiệp thì không nhất thiết cần nhiều về vốn, họ có thể kêu gọi các nguồn tài trợ thông qua từng dự án công việc. Tuy nhiên, để sử dụng nguồn tài chính khi khởi nghiệp một cách cụ thể thì sinh viên cần có kế hoạch chủ động tìm kiếm được nguồn tài chính từ các chủ đầu tư và quỹ hỗ trợ. Để tạo được nguồn vốn khởi nghiệp thì cơ quan quản lý đóng một vai trò quan trọng để sinh viên có thể tiếp cận nguồn vốn bằng nhiều cách như: giảm lãi suất cho vay, phê duyệt dự án nhanh. Đồng thời, nhà trường nên có chính sách để hỗ trợ các hoạt động, các ý tưởng, dự án khởi nghiệp của học sinh, sinh viên trong trường; đồng thời xây dựng quỹ hỗ trợ khởi nghiệp từ nguồn kinh phí xã hội hóa. Hơn nữa, nhà trường nên xây dựng các vườn ươm doanh nghiệp, Trung tâm hỗ trợ khởi nghiệp, xây dựng chương trình, chính sách hỗ trợ hoạt động khởi nghiệp đổi mới sáng tạo... để tư vấn cho sinh viên có ý định khởi nghiệp.

Hạn chế của đề tài và hướng nghiên cứu tiếp theo: số lượng cỡ mẫu còn hạn chế khi sử dụng mô hình cấu trúc tuyến tính (SEM), không so sánh được sự biến đổi từ lúc hình thành ý định đến khi xảy ra hành vi thực tế. Ngoài ra, còn nhiều nhân tố khác tác động đến ý định khởi nghiệp của sinh viên. Hơn nữa, nghiên cứu mới chỉ thực hiện ở 02 Khoa của DNC nên nghiên cứu sau này cần tăng số cỡ mẫu, khảo sát ở các Khoa khác, đồng thời đưa thêm những nhân tố mới vào mô hình nghiên cứu. 


\section{Tài liệu tham khảo}

Ajzen, I. (1991). The theory of planned behavior. Organizational Behavior and Human decision Processes, 50(2), 179-211. doi:10.1016/0749-5978(91)90020-T

Ajzen, I., \& Fishbein, M. (1975). A Bayesian analysis of attribution processes. Psychological Bulletin, 82(2), 261-277.

Anderson, J. C., \& Gerbing, D. W. (1988). Structural equation modeling in practice: A review and recommended two-step approach. Psychological Bulletin, 103(3), 411-423.

Bandura, A. (1997). Self-efficacy: The excercise of control. New York, NY: Freeman.

Bộ Giáo dục và Đào tạo. (2018). Quyết định số 1230/QĐ-BGDĐT ngày 30 tháng 3 năm 2018 về việc ban hành Kế hoạch triển khai đề án "Hỗ trọ" học sinh sinh viên khởi nghiệp đến năm 2025"[Decision No. 1230/QD-BGDĐT dated March 30, 2018 on the issuance of the implementation plan of the project "Supporting students to start a business by 2025"]. Retrieved May 10, 2021, from https: //thuvienphapluat.vn/van-ban/Doanh-nghiep/Quyetdinh-1230-QD-BGDDT-2018-De-an-Ho-tro-hoc-sinh-sinh-vien-khoi-nghiep-78930.aspx

Boissin, J.-P., Branchet, B., Emin, S., \& Herbert, J. I. (2009). Students and entrepreneurship: A comparative study of France and the United States. Journal of Small Business \& Entrepreneurship, 22(2), 101-122. doi:10.1080/08276331.2009.10593445

Bui, D. H. T., Le, L. T., Dao, D. T. X., \& Nguyen, H. T. (2011). Nghiên cứu ảnh hưởng của các yếu tố tính cách cá nhân lên tiềm năng khởi nghiệp của sinh viên [Study the effects of individual personality factors on students' entrepreneurial potential]. Tạp chí Phát triến Khoa học và Công nghệ Đại học Quốc gia TP. Hồ Chí Minh, 14(3Q), 68-82.

Chau, T. T. N., \& Huynh, T. L. T. (2020). Các yếu tố ảnh hưởng đến ý định khởi nghiệp của sinh viên Trường Đại học An Giang [Factors affecting the intention to start a business of An Giang University students]. Tạp chí Công Thuơng, 17.

Comrey, A., \& Lee, H. (1992). A first course in factor analysis. Hillsdale, NJ: Lawrence Eribaum Associates. Inc..

Dong Nghi \& Thien Minh (2018). Doanh nghiệp khởi nghiệp: Vẫn còn rào cản! [Start-ups: Still Barriers!]. Retrieved March 10, 2021, from https://petrotimes.vn/doanh-nghiep-khoinghiep-van-con-rao-can-502524.html

Gupta, V. K., \& Bhawe, N. M. (2007). The influence of proactive personality and stereotype threat on women's entrepreneurial intentions. Journal of Leadership \& Organizational Studies, 13(4), 73-85.

Hair, J. F., Anderson, R., Tatham, R., \& Black, W. (1998). Multivariate data analysis (5th ed.). Upper Saddle River, NJ: Pearson Prentice Hall.

Hair, J. F., Black, W. C., Babin, B. J., Anderson, R. E., \& Tatham, R. (2006). Multivariate data analysis (6th ed.). Upper Saddle River, NJ: Pearson Prentice Hall.

Hoang, T., \& Chu, N. N. M. (2008). Phân tích dũ liệu nghiên cứu với SPSS, tập 2 [Analyze research data with SPSS, volume 2]. Ho Chi Minh, Vietnam: Nhà xuất bản Hồng Đức.

Hooper, D., Coughlan, J., \& Mullen, M. (2008). Structural equation modelling: Guidelines for determining model fit. Journal of Business Research Methods, 6(1), 53-60.

Koe, W.-L. (2016). The relationship between Individual Entrepreneurial Orientation (IEO) and entrepreneurial intention. Journal of Global Entrepreneurship Research, 6(1), 1-13. 
Kolvereid, L. (1996). Prediction of employment status choice intentions. Entrepreneurship Theory and Practice, 21(1), 47-58.

Krueger, N. F., Jr., \& Brazeal, D. V. (1994). Entrepreneurial potential and potential entrepreneurs. Entrepreneurship Theory and Practice, 18(3), 91-104.

Krueger, N. F., Jr., Reilly, M. D., \& Carsrud, A. L. (2000). Competing models of entrepreneurial intentions. Journal of Business Venturing, 15(5/6), 411-432. doi:10.1016/S0883-9026(98)00033-0

Kuckertz, A., \& Wagner, M. (2010). The influence of sustainability orientation on entrepreneurial intentions-Investigating the role of business experience. Journal of Business Venturing, 25(5), 524-539. doi:10.1016/j.jbusvent.2009.09.001

Le, D. T. T., \& Nguyen, A. T. P. (2016). Các nhân tố tác động đến ý định khởi nghiệp của sinh viên thuộc khối ngành kinh tế và kỹ thuật tại Trường Đại học Lạc Hồng [Factors affecting the intention to start a business of students in the field of economics and engineering at Lac Hong University]. Tạp chí Khoa họ Lạc Hồng, 5(62), 83-88.

Le, L. K. (2018). Các nhân tố ảnh hưởng đến ý định khởi nghiệp của sinh viên khối ngành kinh tế tại thành phố Hồ Chí Minh [Factors affecting the intention to start a business of economics students in Ho Chi Minh City]. Tạp chí Phát triển nhân lục, 1(6), 12-24.

Le, Q. (2007). Nghiên cứu quá trình quyết định khởi nghiệp của doanh nhân trẻ Việt Nam- thành phố Hồ Chí Minh [Researching the process of starting a business decision of young Vietnamese entrepreneurs - Ho Chi Minh City]. Tạp chí Phát triển kinh tế, 7.

Le, T. N. D., \& Nguyen, L. M. (2019). Các nhân tố ảnh hưởng đến ý định khởi nghiệp của sinh viên khoa Quản trị kinh doanh [Factors affecting the intention to start a business of students of the Faculty of Business Administration]. Tạp chí khoa hoc Truờng Đại hoc Cửu Long, $16 \& 17,24-35$.

Likert, R. (1932). A technique for the measurement of attitudes. Archives of Psychology, 140, 5-53.

Liñán, F., \& Chen, Y.-W. (2006). Testing the entrepreneurial intention model on a two-country sample (Working Papers 0607). Departament d'Economia de l'Empresa, Universitat Autònoma de Barcelona Document de Treball núm.

Matlay, H., Dinis, A., do Paço, A., Ferreira, J., Raposo, M., \& Rodrigues, R. G. (2013). Psychological characteristics and entrepreneurial intentions among secondary students. Education and Training, 55(8/9), 763-780.

My Thanh (2017). Nỗ lục nhân đôi số doanh nghiệp hiện có [Efforts to double the number of existing businesses]. Retrieved March 13, 2021, from https://baocantho.com.vn/no-lucnhan-doi-so-doanh-nghiep-hien-co-a91035.html

Ngo, T. T. T., \& Cao, V. Q. (2016). Tổng quan lý thuyết về ý định khởi nghiệp của sinh viên [Theoretical overview of students' entrepreneurial intentions]. Tạp chí Khoa hoc Truờng Đại học Mỏ̉, Kinh tế và Quản trị Kinh doanh, 50(5), 56-65.

Nguyen, H. T., \& Nguyen, P. T. K. (2016). Những nhân tố ảnh hưởng đến ý định khởi sự doanh nghiệp của sinh viên Trường Đại học Trà Vinh [Factors affecting the intention to start a business of students at Tra Vinh University]. Tạp chí khoa hoc Truòng Đại học Trà Vinh, $23,1-9$.

Nguyen, T. D. (2011). Phương pháp nghiên cúu khoa học trong kinh doanh [Scientific research methods in business]. Ho Chi Minh, Vietnam: Nhà xuất bản Lao động-Xã hội. 
Ooi, Y. K., Selvarajah, C., \& Meyer, D. (2011). Inclination towardsentrepreneurship among university students: An empirical study of Malaysian university students. International Journal of Business and Social Social Science, 2(4), 206-220.

Phan, T. A., \& Tran, H. Q. (2017). Phân tích các yếu tố ảnh hưởng đến ý định khởi nghiệp kinh doanh của sinh viên Trường Đại học Kỹ thuật Công nghệ Cần Thơ [Analysis of factors affecting the intention to start a business of students at Can Tho University of Technology and Technology]. Tạp chí Khoa học Trương Đại hoc Cần Tho; 48, 96-103.

Phan, V. Q., \& Trac, H. A. (2020). Các yếu tố ảnh hưởng đến ý định khởi nghiệp của sinh viên tại Trường Đại học Phan Thiết, tỉnh Bình Thuận [Factors affecting the intention to start a business of students at Phan Thiet University, Binh Thuan province]. Tạp chí Công Thuong, 18.

Schumacker, R. E., \& Lomax, R. G. (2006). Application of structural equation modeling in educational research and practice. Mahwah, NJ: Lawrence Erlbaum.

Schwarz, E. J., Wdowiak, M. A., Almer- Jarz, D. A., \& Breitenecker, R. J. (2009). The effects of attitudes and perceived environment conditions on students' entrepreneurial intent. Education and Training, 51(4), 272-291. doi:10.1108/00400910910964566

Scott, J. W. (1991). The evidence of experience. Critical Inquiry, 17(4), 773-797.

Shapero, A., \& Sokol, L. (1982). The social dimensions of entrepreneurship. In C. Kent, L. Sexton \& K. Vesper (Eds.), Encylopedia of Entrepreneurship, 72-90. Englewood Cliffs, NJ: Prentice Hall.

Souitaris, V., Zerbinati, S., \& Al-Laham, A. (2007). Do entrepreneurship programmes raise entrepreneurial intention of science and engineering students? The effect of learning, inspiration and resources. Journal of Business Venturing, 22(4), 566-591. doi:10.1016/j.jbusvent.2006.05.002

Steenkamp, J.-B. E., \& Van Trijp, H. C. (1991). The use of LISREL in validating marketing constructs. International Journal of Research in Marketing, 8(4), 283-299.

Taylor, S. A., Sharland, A., Cronin, J. J., \& Bullard, W. (1993). Recreational service quality in the international setting. International Journal of Service Industry Management, 4(4), 68-86.

Thu Hang (2020). Có thể mất 15 triệu co hội việc làm dành cho thanh niên trong năm 2020 [Could lose 15 million job opportunities for young people in 2020]. Retrieved March 13, 2021, from https://thanhnien.vn/gioi-tre/co-the-mat-15-trieu-co-hoi-viec-lam-danh-cho-thanh-nientrong-nam-2020-1267323.html

Truong, T. D., \& Nguyen, L. T. T. (2019). Một số nhân tố tác động đến ý định khởi nghiệp của sinh viên [Some factors affecting students' intention to start a business]. Tạp chí Công Thwong, 3, 99-104.

Vojak, B. A., Griffin, A., Price, R. L., \& Perlov, K. (2006). Characteristics of technical visionaries as perceived by American and British industrial physicists. R\&D Management, 36(1), 17-26.

Wilbard, F. (2009). Entrepreneurship proclivity: An exploratory study on students' entrepreneurship intention (Master's thesis). University of Agder, Kristiansand, Norway.

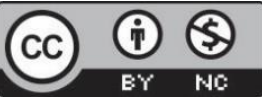

Creative Commons Attribution-NonCommercial 4.0 International License. 